\title{
Drug Resistance Patterns and Virus Re-Suppression among HIV-1 Subtype C Infected Patients Receiving Non-Nucleoside Reverse Transcriptase Inhibitors in South Africa
} \author{
Max Petzold ${ }^{6}$, Lynn Morris ${ }^{2}$ and Rami Kantor ${ }^{7}$ \\ ${ }^{1}$ Division of Global Health (IHCAR), Karolinska Institutet, Stockholm, Sweden \\ ${ }^{2}$ AIDS Virus Research Unit, National Institute for Communicable Diseases (NICD), Johannesburg, South Africa \\ ${ }^{3}$ Center for Statistical Sciences, Brown University, Providence, Rhode Island, USA \\ ${ }^{4}$ Division of Infectious Diseases, Stanford University, California, USA \\ ${ }^{5}$ Perinatal HIV Research Unit (PHRU), University of the Witwatersrand, Soweto, South Africa \\ ${ }^{6}$ Nordic School of Public Health (NHV), Gothenburg, Sweden \\ ${ }^{7}$ Division of Infectious Diseases, Brown University Alpert Medical School, Providence, Rhode Island, USA
}

Ziad El-Khatib ${ }^{1,2 *}$, Allison K. DeLong ${ }^{3}$, David Katzenstein ${ }^{4}$, Anna Mia Ekstrom¹, Johanna Ledwaba ${ }^{2}$, Lerato Mohapi ${ }^{5}$, Fatima Laher ${ }^{5}$,

\begin{abstract}
Background: Emergence of HIV-1 drug resistance is at times an inevitable and anticipated consequence of antiretroviral therapy (ART) failure. We examined drug resistance patterns and virus re-suppression among subtype C-infected South African patients receiving first-line ART.

Methods: Treatment records of 431 patients on NNRTI-containing regimens for a median of 45 months were analyzed. Patients with viral load (VL) $>400$ copies $/ \mathrm{mL}$ were followed and drug resistance mutations (DRM) were re-assessed. Associations between clinical/demographic measures and drug resistance/virologic outcomes were examined using Fisher exact and ordinal and logistic regression.

Results: Ten percent of patients (43/431) were viremic at enrollment $(98 \%)$ sequences were obtained from 38/43. Of those, $82 \%$ had 1-7 DRM. In bivariate analysis remote exposure to single-dose nevirapine or prior ART; higher CD4 counts; lower VL; and >6 months of virologic failure were significantly associated with number of DRM. Of 25 viremic patients followed for a median of 8 months on a continued first-line regimen, 12 (48\%) re-suppressed, six with $\mathrm{K} 103 \mathrm{~N}$ and three with M184V. Thirteen $(52 \%)$ had continued virologic failure which was significantly associated with detectable $V L>6$ months prior to enrollment and number of DRM.

Conclusion: Among these HIV-1 subtype C-infected patients, DRM numbers and patterns were associated with prior exposure to sub-optimal ART, adherence and duration of virologic failure. Viral re-suppression in the presence of $\mathrm{K} 103 \mathrm{~N}$ and M184V challenges assumptions about drug resistance. In resource-limited settings, where genotyping and alternative drug options are unavailable, continuing first-line treatment, reinforcing adherence and regular virologic monitoring may be effective even after virologic failure.
\end{abstract}

Keywords: HIV-1; Subtype C; Drug resistance; Mutations; NNRTI; First-line ART; South Africa; ART experienced

\section{Background}

More than 1 million HIV-1 subtype C infected patients in South Africa are receiving antiretroviral therapy (ART) [43]. In 2004 a national treatment program was initiated, including a first-line regimen containing a non-nucleoside reverse transcriptase inhibitor (NNRTI), either efavirenz or nevirapine, in combination with NRTI, stavudine or zidovudine, and lamivudine [27] The virologic outcomes of firstline regimens among subtype C-infected people in South Africa are comparable to those among subtype B infected patients in Switzerland, where approximately $10 \%$ of patients experience virologic failure after 12 months and up to $25 \%$ experience virologic failure by two years on ART [20].

Risk factors contributing to virologic failure and drug resistance in sub-Saharan Africa include incomplete adherence [1,11,30,36], treatment interruptions $[39,41]$, low CD4 cell counts $[14,15,21,41]$ low body weight before ART initiation [21] and prior exposure to single dose nevirapine (sdNVP) for prevention of mother-to-child transmission (pMTCT) and/or dual nucleoside treatment $[6,22,25]$. The majority $(>80 \%)$ of viremic patients harbor drug resistance mutations (DRM) $[2,11,14,28,33]$ and maintaining a failing ART regimen can lead to accumulation of DRM $[14,21,32]$ and increased ART cross-resistance

\section{$[5,18]$}

Five recent Southern African studies among NNRTI recipients identified treatment failure by virologic or immunologic criteria $[11,14,28,33,42]$. The prevalence of DRM ranged from $62 \%$ to $95 \%$ $[14,15,28,33,42]$. In the first year of treatment Marconi et al. [28] in KwaZulu-Natal $(n=115)$ and Orrell et al. [33] in Cape Town $(n=110)$, identified DRM among $83 \%$ and $>87 \%$ respectively. In longer term studies, Hoffmann et al. [3,14] in South Africa $(n=68)$ and Hosseinipour et al. [15] in Malawi ( $\mathrm{n}=94)$ reported DRM after a median $>36$ months among $62 \%$, and 95\% respectively. Wallis et al. [42] in Johannesburg

*Corresponding author: Ziad El-Khatib, Division of Global Health (IHCAR), Nobels 9, Karolinska Institutet, SE-171 77 Stockholm, Sweden, Tel: +46-73-776-4859; Fax: +46-8-311590; E-mail: ziad.khatib@gmail.com

Received January 12, 2011; Accepted February 15, 2011; Published February 18, 2011

Citation: El-Khatib Z, DeLong AK, Katzenstein D, Ekstrom AM, Ledwaba J, et al (2011) Drug Resistance Patterns and Virus Re-Suppression among HIV-1 Subtype C Infected Patients Receiving Non-Nucleoside Reverse Transcriptase Inhibitors in South Africa. J AIDS Clinic Res 2:117. doi:10.4172/2155-6113.1000117

Copyright: (c) 2011 El-Khatib Z, et al. This is an open-access article distributed under the terms of the Creative Commons Attribution License, which permits unrestricted use, distribution, and reproduction in any medium, provided the original author and source are credited. 
Citation: El-Khatib Z, DeLong AK, Katzenstein D, Ekstrom AM, Ledwaba J, et al. (2011) Drug Resistance Patterns and Virus Re-Suppression among HIV-1 Subtype C Infected Patients Receiving Non-Nucleoside Reverse Transcriptase Inhibitors in South Africa. J AIDS Clinic Res 2:117. doi:10.4172/2155-6113.1000117

Page 2 of 11

reported DRM among $84 \%$ of 226 viremic patients, however duration of treatment was not reported.

Previously [11], we surveyed viremia and drug resistance prevalence among 998 patients in Soweto, South Africa and found that $94 / 883$ (11\%) receiving first-line regimens for a median of 42 months were viremic and 78/94 (83\%) had drug resistance. Here, we obtained retrospective data on 431 of these patients, enrolled at a single clinic, and examined factors associated with the evolution and patterns of DRM. Additionally, we followed 25 of these viremic patients to explore the implications of DRM on continued NNRTI-based treatment.

\section{Methods}

\section{Study sites and patients}

The study was done at the Perinatal HIV Research Unit (PHRU), a non-governmental organization (NGO) research clinic in Chris Hani Baragwanath Hospital, Soweto, outside Johannesburg, South Africa(11, 29). At the time of the study, the clinic staff consisted of five medical doctors, two nursing assistants and two counselors managing around 1500 ART recipients, with 50 daily visits [11].

Consenting patients were enrolled from March through September 2008 if they were $\geq 18$ years old and $>12$ months on a first-line regimen [11]. At study enrollment, viral load (VL), CD4 cell count and HIV1 reverse transcriptase (RT) genotype were assessed and basic demographic information obtained. Virologic failure was defined as $\mathrm{VL}>400$ copies $/ \mathrm{mL}$ at study enrollment [11]. Persistent virologic failure and re-suppression were defined as $\mathrm{VL}>400$ copies/ $\mathrm{mL}$ and return to $\leq$ 400 copies/mL, respectively, at follow-up (Figure 1).

Medical records were reviewed retrospectively to extract information on potential risk factors that may be associated with DRM or re-suppression, including age, gender, year of HIV diagnosis, ART regimens and dates, history of sdNVP or other ART exposure, tuberculosis (TB) treatment, pre-ART initiation VL and CD4 cell counts, WHO stage prior to ART initiation and any treatment interruptions in the last six months prior to study enrollment. Virologic
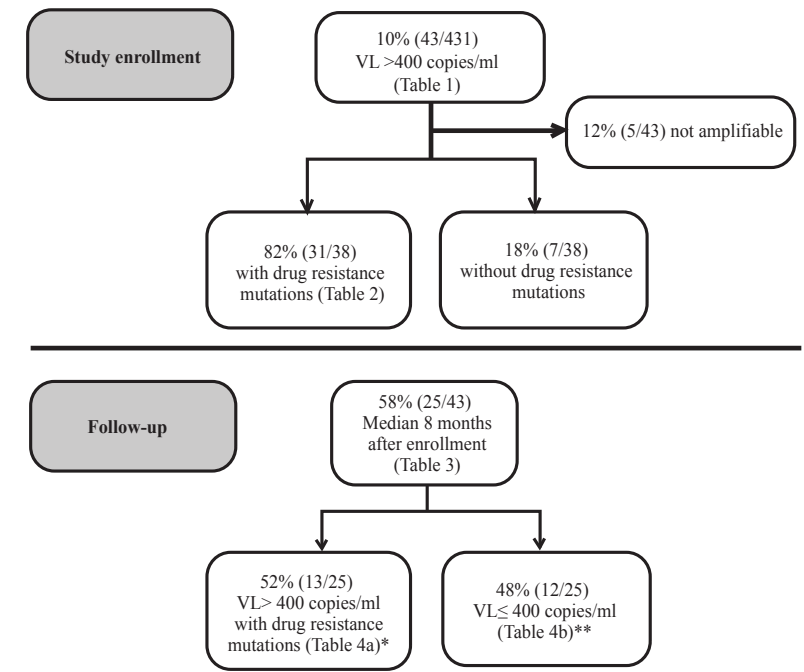

* At study enrollment: 1/13 wild type and 1/13: not amplifiable ${ }^{*}$ At study enrollment 2/12 wild type and 3/12: not amplifiable

$\mathrm{VL}$, virus load failure prior to study enrollment, was defined as VL $>400$ copies $/ \mathrm{mL}$ at either of the prior two visits in the last 12 months. Poor adherence was considered to be returning more than seven days late for the drug refill appointment pre-study enrollment. An instrument was designed and tested to extract information from medical records using Epi Data $[9,10]$. The study and consent forms were approved by the University of the Witwatersrand Human Research Ethics Committee in South Africa and the Regional Medical Ethics Board in Stockholm, Sweden.

\section{Laboratory assessments}

CD4 cell counts were performed by FACSCountTM (Becton Dickinson BioSciences, Immunocytochemistry Systems, San Jose, California, USA) and VL was measured using the Roche Amplicor, version 1.5 (Roche Molecular Diagnostics, Basel, Switzerland) with a lower limit of detection of 400 copies $/ \mathrm{mL}$. Both assays were performed at the National Institute for Communicable Diseases (NICD), Johannesburg. VL data extracted from medical records that were done as part of routine clinical care used the Versant HIV-1 RNA 3.0 (Siemens Deerfield, IL, USA) bDNA technology. For HIV-1 drug resistance testing, an in-house genotyping assay was performed at the NICD [35].

\section{Drug resistance mutations and susceptibility scoring}

Mutations were identified by the Stanford HIVdb genotypic resistance algorithm [13] and coded as major DRM as defined by the International AIDS Society (IAS) December 2009 list [16]. Subtype was established using the Rega subtyping tool v.2.0, which incorporates rigorous phylogenetic analyses [7]. Sequence quality was confirmed prior to analysis by[18] inspecting sequences for possible frame shifts, high numbers of ambiguous nucleic and/or amino acids, extreme levels of pair-wise genetic distances, and unique amino acids or stop codons. To predict phenotypic drug resistance the Stanford HIV database (HIVdb) scoring system was applied [13] and a resistance score calculated as (i) susceptible (0-9) to potentially low-level (10-14); (ii) low (15-29); (iii) intermediate (30-59); and (iv) high level of resistance (score $\geq 60$ ).

\section{Data analysis and statistics}

Risk factors were examined for associations with two drug resistance outcomes: (i) number of all-class DRM at enrollment; and (ii) continued viremia versus re-suppression at follow-up. Associations between viral re-suppression and presence of any DRM, number of NRTI and NNRTI DRM and the total number of DRM at enrollment were examined. Due to the small sample size, bivariate analysis was performed without adjusting for confounding variables and the results must be interpreted with this in mind.

Ordinal logistic regression was used to examine the association between risk factors and the number of DRM at enrollment expressed as odds ratios (OR), and 95\% confidence intervals (95\% CI). Unlike Poisson regression, ordinal logistic regression can be fitted to zeroinflated data and does not assume that the events (i.e. accumulation of DRM) are independent and occur at a constant rate. Each model was checked to ensure the assumption of proportional odds between successive DRM categories was met. To examine risk factors associated with persistent virologic failure at follow-up, Fisher exact tests were used for categorical risk factors (OR, 95\% CI) and Wilcoxon rank sum tests for continuous risk factors (difference in median, 95\% CI). Analysis was performed using Stata/SE College Station, Texas (version 10.1) (38) and $R$ (version 2.11.1) (40). P-values less than 0.05 were considered statistically significant. 
Citation: El-Khatib Z, DeLong AK, Katzenstein D, Ekstrom AM, Ledwaba J, et al. (2011) Drug Resistance Patterns and Virus Re-Suppression among HIV-1 Subtype C Infected Patients Receiving Non-Nucleoside Reverse Transcriptase Inhibitors in South Africa. J AIDS Clinic Res 2:117. doi:10.4172/2155-6113.1000117

Page 3 of 11

\section{Results}

\section{Patient characteristics}

(Table 1) displays characteristics of the 431 patients who had been on ART for at least 12 months where $75 \%$ were females, $96 \%$ were born in South Africa, $90 \%$ had above primary school education and the median age at study enrollment was 38 years. Ninety-one percent were receiving efavirenz-based therapy and $9 \%$ a nevirapine-based therapy.

Before ART initiation, the median VL and CD4 were 71,995 (range 1,078 to $>500,000$ ) copies/mL and 93 (range 1 to 444 ) cells/ mm3 respectively. At study enrollment, patients had received ART for a median of 45 months (range 13 to 152) and the CD4 cell count increased to 419 (range 16 to 1,270 ).

Forty-three patients (10\%) had VL>400 copies/mL (median 6,510; range 407 to $>500,000)$ at enrollment, almost all $(98 \%)$ previously suppressed on ART. Median time on ART was similar among patients with $\mathrm{VL}<$ and $>400$ copies $/ \mathrm{mL}$ ( $\mathrm{p}=0.86$ ). However at study enrollment, those with $\mathrm{VL}>400$ copies/mL had a significantly lower CD4 cell count compared to patients with $\mathrm{VL}<400$ copies $/ \mathrm{mL}(\mathrm{p}<0.01)$.

Among 40/43 patients for whom there was a record of previous ART exposure: $8 / 31$ (26\%) females had received sdNVP prior to initiating ART and 5/40 (13\%) patients had been exposed to other ART before initiating the current ART regimen. Compared to unexposed individuals, there was a borderline association between viremia at study enrollment and exposure to sdNVP $(\mathrm{p}=0.05)$.

\section{Drug resistance mutations at study enrollment}

Thirty-eight of 43 samples were successfully genotyped; 31/38 (82\%) had at least one DRM and 24 (63\%) had $\geq 3$ DRM (Table 2). Of the genotyped samples 5/38 (13\%) had K103N alone; $2 / 38$ (5\%) had M184V and K103N; and 10/38 (26\%) had three mutations with M184V/I, K103N and an additional NNRTI mutation. Finally $14 / 38$ (37\%) had $\geq 4$ mutations: $13 / 14$ (93\%) M184V/I and 8/14 (57\%) K103N, all with $\geq 2$ NNRTI and most with one or more thymidine analogue mutations (TAM) or other NRTI mutations. Overall, $8 / 38(21 \%)$ had one or more TAMs, three had A62V or V75I and only one patient had K65R.

Several risk factors were significantly associated with increased numbers of DRM (Table 3). Patients with prior exposure to either sdNVP or other ART had more mutations than those not previously exposed. The ordinal regression OR was 3.8 (95\%CI 1.1 to $15.2 ; \mathrm{p}=0.03)$, i.e. it was 3.8 times more likely for patients with prior ART exposure to have $\geq 1$ vs. $0, \geq 2$ vs. $\leq 1, \geq 3$ vs. $\leq 2$ DRM and so on. Number of DRM was positively associated with being female (OR 5.6; 95\%CI 1.3 to 24.5 ; $\mathrm{p}=0.02$ ), having a higher CD4 cell count (OR 1.7 per 100 CD 4 cells; 95\% CI 1.1 to 2.7; $\mathrm{p}=0.02$ ) and having detectable $\mathrm{VL}$ at one of two earlier scheduled visits (OR $8.4 ; 95 \% \mathrm{CI} 1.9$ to $42.4 ; \mathrm{p}<0.01$ ). The association with gender was mainly explained by prior exposure to pMTCT, mainly sdNVP, among the women. Only one male had any prior exposure to ART. The number of DRM was negatively associated with coming late for the drug refill visit in the last month (OR $0.1 ; 95 \% \mathrm{CI} 0$ to 0.5 ; $\mathrm{p}=0.01$ ) and with VL such that for participants with 1-log unit higher VL the odds of having a higher number of DRM was 0.5 (95\%CI 0.2 to $1.0 ; \mathrm{p}=0.04$ ). Finally the median VL of the seven patients with no DRM was 83,000 copies $/ \mathrm{mL}$ compared with a median VL of 6,510 copies $/ \mathrm{mL}$ among those with at least one DRM, providing evidence for existing but incomplete drug pressure amongst those with DRM.

\section{Risk factors for persistent virologic failure and drug resistance mutations at follow-up}

Follow-up data and samples were available for 25/43 (58\%) of the viremic patients, after a median of 8 (range 4 to 10) months (Figure 1). Persistent virologic failure at follow-up, in $13 / 25$ (52\%) patients, was associated with a detectable VL in the two visits prior to study enrollment $(\mathrm{p}<0.01)$ and the number of DRM at study enrollment (OR 2.36; 95\%CI 1.11 to $5.02 ; \mathrm{p}=0.04$ ), particularly NRTI mutations (OR 3.68; 95\%CI 1.11 to 12.17; $\mathrm{p}=0.05$ ) (Table 3). All 13 patients had genotypic resistance with six additional DRM acquired at follow-up, leading to high level predicted resistance to efavirenz and/or nevirapine $(100 \%)$ and lamivudine $(100 \%)$ and intermediate to low predicted resistance to etravirine in $7 / 13$ (54\%) (Table 4a). Although viremic, patient number 35 did not have any DRM at study enrollment, but with continued treatment and presumably better adherence, three DRM were selected at follow-up with a persistent, albeit lower, VL for nine months. At study enrollment nine of these patients had failed first-line regimens with full predicted susceptibility to NRTIs.

Twelve of the 25 (48\%) patients re-suppressed at follow-up, after a median of eight additional months on treatment with the same NNRTI (mostly efavirenz)-based regimen. Comparison of clinical and laboratory characteristics of these 12 patients with the 13 who had persistent virologic failure showed no significant differences in sex, median CD4 and VL prior to ART initiation. At study enrollment $3 / 12(25 \%)$ re-suppressed patients could not be amplified and three (25\%) had no DRM. However, the remaining six patients who were resuppressed had NNRTI DRM, three had K103N, one had K103N and M184V and one had K103N, V106M and M184V (Table 4b). The sixth patient had three NNRTI and 3 NRTI mutations. Thus, six patients with high level NNRTI resistance and three patients with high level NNRTI and lamivudine resistance achieved re-suppression while continuing the same first-line regimen.

\section{Discussion}

HIV-1 drug resistance is a potential cause and is often a consequence of virologic failure. In this study, we examined drug resistance in HIV1 subtype $\mathrm{C}$ infected South African patients failing first-line regimens after a minimum of 12 months on ART. Of 43 long-term ART recipients with viremia, most had multiple DRM. Among 25 of these patients followed on continued NNRTI-based ART, 12 achieved virologic suppression and 13 had persistent VL $>400$ copies/mL. We examined the characteristics and patterns of DRM, the estimated drug resistance and implications for further therapies among these ART experienced, subtype $\mathrm{C}$ infected patients.

In examination of co-occurrence of DRM, $82 \%$ of 38 available RT sequences demonstrated a wide spectrum (range 1 to 7 ) of cooccurring, DRM. The majority (63\%) had $\geq 3$ DRM, mostly including K103N accompanied by M184V/I, at times with complex mixtures of additional NRTI and NNRTI mutations. Patients with prior ART drug exposure and those with detectable VL and higher CD4 cell counts prior to or at study enrollment, respectively, tended to have a higher number of DRM. These results are likely to reflect the selection of drug resistance due to lapses in adherence, reduced drug exposure and inadequate drug pressure. Conversely, patients who were late to pharmacy or who had higher VL at study enrollment tended to have fewer DRM, perhaps reflecting a very low adherence.

Drug resistance mutations among subtype C NNRTI recipients have been identified after virologic or immunologic failure based on 
Citation: El-Khatib Z, DeLong AK, Katzenstein D, Ekstrom AM, Ledwaba J, et al. (2011) Drug Resistance Patterns and Virus Re-Suppression among HIV-1 Subtype C Infected Patients Receiving Non-Nucleoside Reverse Transcriptase Inhibitors in South Africa. J AIDS Clinic Res 2:117. doi:10.4172/2155-6113.1000117

Page 4 of 11

\begin{tabular}{|c|c|c|c|c|}
\hline & $\begin{array}{l}\text { All patients } \\
\mathrm{N}=431\end{array}$ & $\begin{array}{l}\text { Suppressed at study enroll- } \\
\text { ment } \mathrm{N}=388\end{array}$ & $\begin{array}{l}\text { Viremic at study enrollment } \\
\qquad \mathrm{N}=43\end{array}$ & $p$ value \\
\hline Female & $325(75 \%)$ & $291(75 \%)$ & $34(79 \%)$ & 0.56 \\
\hline Age, median in years (range) & $38(21,64)$ & $38(21,64)$ & $36(25,60)$ & 0.35 \\
\hline Born in South Africa & $414(96 \%)$ & $373(96 \%)$ & $41(95 \%)$ & 0.80 \\
\hline \multicolumn{5}{|l|}{ Education } \\
\hline Never been to school & $7(2 \%)$ & $6(2 \%)$ & $1(2 \%)$ & 0.64 \\
\hline Primary school & $42(10 \%)$ & $39(10 \%)$ & $3(7 \%)$ & \\
\hline Secondary school & $357(83 \%)$ & $319(82 \%)$ & $38(88 \%)$ & \\
\hline Tertiary school & $25(6 \%)$ & $24(6 \%)$ & $1(2 \%)$ & \\
\hline \multicolumn{5}{|l|}{ In any form of a relationshipa } \\
\hline Yes & $250(58 \%)$ & $227(59 \%)$ & $23(54 \%)$ & \\
\hline No & $181(42 \%)$ & $161(41 \%)$ & $20(46 \%)$ & 0.53 \\
\hline $\begin{array}{l}\text { Months on ART, at study enrollment } \\
\text { (range) }\end{array}$ & $45(13,152)$ & $45(13,152)$ & $45(13,55)$ & 0.86 \\
\hline \multicolumn{5}{|l|}{ ART regimen } \\
\hline Efavirenz containingb & $393(91 \%)$ & $357(92 \%)$ & $36(94 \%)$ & \\
\hline Stavudine containingc & $254(59 \%)$ & $225(58 \%)$ & $29(67 \%)$ & 0.35 \\
\hline \multicolumn{5}{|l|}{ VL prior to starting ART } \\
\hline Median copies/mL (range)d & $70870(1078,>500000)$ & $67315(2$ 102, >500 000) & $86718(1078,>500000)$ & 0.48 \\
\hline \multicolumn{5}{|l|}{ CD4 prior to starting ART } \\
\hline Median cells/mm3 (range) & $93(1,444)$ & $95(1,760)$ & $107(6,314)$ & 0.34 \\
\hline Mean & 96 & 104 & 109 & 0.74 \\
\hline \multicolumn{5}{|l|}{ CD4 at study enrollment } \\
\hline Median cells/mm3 (range) & $419(16,1270)$ & $437(119,1270)$ & $276(16,642)$ & $<0.01$ \\
\hline \multicolumn{5}{|l|}{ Prior exposure to ART } \\
\hline No & $335(81 \%)$ & $308(82 \%)$ & $27(68 \%)$ & \\
\hline PMTCT only & $45(11 \%)$ & $37(10 \%)$ & $8(20 \%)$ & $0.05 f$ \\
\hline Othere & $35(8 \%)$ & $30(8 \%)$ & $5(13 \%)$ & 0.21 \\
\hline $\begin{array}{l}\text { Exposure to sdNVP or other ART vs. no } \\
\text { exposuree }\end{array}$ & $80(19 \%)$ & $67(18 \%)$ & $13(32 \%)$ & $0.03 f$ \\
\hline \multicolumn{5}{|l|}{ WHO stage prior to starting ART } \\
\hline I & $61(21 \%)$ & $55(20 \%)$ & $6(25 \%)$ & \\
\hline II & $86(29 \%)$ & $82(31 \%)$ & $4(17 \%)$ & $0.32 f$ \\
\hline III & $137(47 \%)$ & $123(46 \%)$ & $14(58 \%)$ & $1.00 f$ \\
\hline IV & $9(3 \%)$ & $9(3 \%)$ & $0(0 \%)$ & --- \\
\hline
\end{tabular}

ART, antiretroviral therapy; VL, virus load; WHO, world health organization, PMTCT, prevention mother-to-child-transmission by administrating a single dose nevirapine; $\mathrm{N}$, number

aYes: cohabitation, married, sexual relationship, No: Single/divorced/separated/widow

${ }^{\mathrm{b}}$ The other drug option was nevirapine

'The other drug option was zidovudine

${ }^{d}$ VL done using Versant HIV-1 RNA 3.0 (Siemens Deerfield, IL, USA) bDNA technology

exposure to other antiretroviral drugs prior to ART initiation

f2-sided Fisher exact test

Table 1: Demographic, clinical and laboratory characteristics of patients

\begin{tabular}{|c|c|c|c|c|c|c|}
\hline $\begin{array}{l}\text { Number of drug resis- } \\
\text { tance mutations } \\
\text { (n pts) }\end{array}$ & Months on ART & Sex & $\begin{array}{l}\text { VL at study } \\
\text { enrollment } \\
\text { (copies } / \mathrm{mL} \text { ) }\end{array}$ & $\begin{array}{l}\text { CD4 at study } \\
\text { enrollment } \\
\text { (cells/mm3) }\end{array}$ & NNRTI & NRTI \\
\hline \multicolumn{7}{|l|}{$\geq 5$ (n=8 pts) } \\
\hline $1^{*}$ & 48 & $\mathrm{~F}$ & 3010 & 524 & $\mathrm{~K} 101 \mathrm{E}+\mathrm{V} 108 \mathrm{I}+\mathrm{Y} 181 \mathrm{C}$ & $\mathrm{D} 67 \mathrm{~N}+\mathrm{K} 70 \mathrm{R}+\mathrm{M} 184 \mathrm{~V}+\mathrm{K} 219 \mathrm{E}$ \\
\hline $2^{* *}$ & 49 & $\mathrm{~F}$ & 2680 & 213 & $\mathrm{~K} 101 \mathrm{H}+\mathrm{K} 103 \mathrm{~N}+\mathrm{V} 106 \mathrm{M}+\mathrm{G} 190 \mathrm{~A}+\mathrm{F} 227 \mathrm{~L}$ & $\mathrm{D} 67 \mathrm{~N}+\mathrm{M} 184 \mathrm{~V}$ \\
\hline $3^{*}, \wedge$ & 41 & $\mathrm{~F}$ & 4310 & 354 & $\mathrm{~K} 101 \mathrm{E}+\mathrm{V} 106 \mathrm{M}+\mathrm{G} 190 \mathrm{~A}$ & $\mathrm{D} 67 \mathrm{~N}+\mathrm{M} 184 \mathrm{~V}$ \\
\hline 4 & 44 & $\mathrm{~F}$ & 75000 & 89 & $\mathrm{~K} 103 \mathrm{~N}+\mathrm{P} 225 \mathrm{H}$ & $\mathrm{D} 67 \mathrm{~N}+\mathrm{K} 70 \mathrm{R}+\mathrm{M} 184 \mathrm{~V}+\mathrm{T} 215 \mathrm{~F}+\mathrm{K} 219 \mathrm{E}$ \\
\hline $5^{*}$ & 22 & $\mathrm{~F}$ & 1850 & 342 & K103N+V108I & $\mathrm{A} 62 \mathrm{~V}+\mathrm{V} 75 \mathrm{I}+\mathrm{M} 184 \mathrm{~V}$ \\
\hline $6^{*}$ & 45 & $\mathrm{~F}$ & 773 & 384 & V106M+G190A & M41L+D67N+K70R+M184V \\
\hline $7^{*}$ & 44 & $\mathrm{~F}$ & 2530 & 199 & V106M+Y188LH & $\mathrm{D} 67 \mathrm{~N}+\mathrm{M} 184 \mathrm{~V}+\mathrm{K} 219 \mathrm{E}$ \\
\hline 8 & 45 & $\mathrm{~F}$ & 18200 & 496 & V106M+Y188L & $\mathrm{M} 41 \mathrm{~L}+\mathrm{D} 67 \mathrm{~N}+\mathrm{K} 70 \mathrm{R}+\mathrm{M} 184 \mathrm{~V}+\mathrm{T} 215 \mathrm{Y}$ \\
\hline \multicolumn{7}{|l|}{4 (n=6 pts) } \\
\hline 9 & 15 & $\mathrm{~F}$ & 16900 & 160 & K101E+V108I+G190A & M184V \\
\hline 10 & 45 & $\mathrm{~F}$ & 11000 & 464 & $\mathrm{~K} 101 \mathrm{H}+\mathrm{K} 103 \mathrm{~N}+\mathrm{G} 190 \mathrm{~A}$ & M184V \\
\hline 11 & 46 & $\mathrm{~F}$ & 3000 & 354 & $\mathrm{~K} 103 \mathrm{~N}+\mathrm{P} 225 \mathrm{H}$ & V75I+M184V \\
\hline
\end{tabular}


Citation: El-Khatib Z, DeLong AK, Katzenstein D, Ekstrom AM, Ledwaba J, et al. (2011) Drug Resistance Patterns and Virus Re-Suppression among HIV-1 Subtype C Infected Patients Receiving Non-Nucleoside Reverse Transcriptase Inhibitors in South Africa. J AIDS Clinic Res 2:117. doi:10.4172/2155-6113.1000117

Page 5 of 11

\begin{tabular}{|c|c|c|c|c|c|c|}
\hline $12^{*}$ & 37 & $M$ & 49900 & 16 & $\mathrm{~K} 103 \mathrm{~N}+\mathrm{Y} 181 \mathrm{C}+\mathrm{P} 225 \mathrm{H}$ & M184I \\
\hline $13^{\wedge}$ & 47 & $\mathrm{~F}$ & 54700 & 419 & $\mathrm{~K} 103 \mathrm{~N}+\mathrm{G} 190 \mathrm{~A}+\mathrm{P} 225 \mathrm{H}$ & M184V \\
\hline 14 & 47 & $\mathrm{~F}$ & 9670 & 377 & $\mathrm{~K} 103 \mathrm{~N}+\mathrm{V} 106 \mathrm{~A}+\mathrm{G} 190 \mathrm{~A}$ & K65R \\
\hline \multicolumn{7}{|c|}{3 (n=10 pts) } \\
\hline $15^{*}$ & 48 & $\mathrm{~F}$ & 18300 & 331 & K101E+Y188L & M184I \\
\hline 16 & 13 & $\mathrm{~F}$ & 6890 & 214 & $\mathrm{~K} 101 \mathrm{E}+\mathrm{V} 106 \mathrm{M}$ & M184V \\
\hline 17 & 41 & $\mathrm{~F}$ & 5340 & 319 & K103N & $\mathrm{D} 67 \mathrm{~N}+\mathrm{M} 184 \mathrm{~V}$ \\
\hline $18^{*}$ & 46 & $\mathrm{~F}$ & 407 & 269 & $\mathrm{~K} 103 \mathrm{~N}+\mathrm{P} 225 \mathrm{H}$ & M184V \\
\hline 19 & 47 & $\mathrm{~F}$ & 6510 & 190 & K103N+V108I & M184V \\
\hline $20^{*}$ & 47 & $\mathrm{~F}$ & 1330 & 277 & $\mathrm{~K} 103 \mathrm{~N}+\mathrm{V} 108 \mathrm{I}$ & M184V \\
\hline 21 & 47 & M & 31000 & 99 & $\mathrm{~K} 103 \mathrm{~N}+\mathrm{V} 108 \mathrm{I}$ & M184V \\
\hline $22^{* *}$ & 47 & $\mathrm{~F}$ & 1280 & 642 & $K 103 N+V 106 M$ & M184V \\
\hline $23^{*}$ & 40 & $\mathrm{~F}$ & 955 & 276 & V106M & $\mathrm{A} 62 \mathrm{~V}+\mathrm{M} 184 \mathrm{~V}$ \\
\hline 24 & 84 & $\mathrm{~F}$ & 43800 & 174 & $\mathrm{~V} 106 \mathrm{M}+\mathrm{Y} 188 \mathrm{C}$ & M184V \\
\hline \multicolumn{7}{|c|}{2 (n=2 pts) } \\
\hline 25 & 43 & $M$ & 430 & 151 & K103N & M184V \\
\hline $26 * \star \wedge$ & 47 & $\mathrm{~F}$ & 1370 & 320 & K103N & M184V \\
\hline \multicolumn{7}{|c|}{1 (n=5 pts) } \\
\hline $27^{* *}$ & 44 & $M$ & 178000 & 47 & K103N & --- \\
\hline $28^{* *}$ & 46 & $\mathrm{~F}$ & 56500 & 187 & K103N & --- \\
\hline $29^{* *}$ & 45 & $\mathrm{~F}$ & 42800 & 100 & K103N & --- \\
\hline $30^{*}$ & 45 & $M$ & 1130 & 439 & K103N & --- \\
\hline $31^{\wedge}$ & 46 & $\mathrm{~F}$ & 43900 & 193 & K103N & --- \\
\hline 32 & 46 & $F$ & 552 & 369 & No major mutations & No major mutations \\
\hline $33^{* *}$ & 41 & $\mathrm{~F}$ & 882000 & 90 & No major mutations & No major mutations \\
\hline $34^{* \star}$ & 47 & $\mathrm{~F}$ & 1450 & 192 & No major mutations & No major mutations \\
\hline $35^{*}, \wedge$ & 43 & M & 83000 & 103 & No major mutations & No major mutations \\
\hline $36^{\wedge}$ & 47 & $\mathrm{~F}$ & 77500 & 232 & No major mutations & No major mutations \\
\hline $37^{* *}, \wedge$ & 43 & $M$ & 312000 & 203 & No major mutations & No major mutations \\
\hline $38^{\wedge}$ & 50 & $M$ & 493000 & 157 & No major mutations & No major mutations \\
\hline \multicolumn{7}{|c|}{ Not amplifiable ( $n=5$ pts) } \\
\hline 39 & 44 & $F$ & 463 & 357 & --- & --- \\
\hline $40^{*}, \wedge$ & 43 & $\mathrm{~F}$ & 4660 & 554 & --- & --- \\
\hline $41^{* *}, \wedge$ & 41 & M & 1060 & 430 & --- & --- \\
\hline $42^{* *}$ & 13 & $\mathrm{~F}$ & 557 & 445 & --- & --- \\
\hline $43^{* \star}$ & 55 & $\mathrm{~F}$ & 66800 & 379 & --- & --- \\
\hline
\end{tabular}

Pts, patients; F, female; M, male; ART, antiretroviral therapy; NRTI, nucleoside reverse transcriptase inhibitor; NNRTI, non-NRTI, VL: virus load; *Persistent virologic failure at follow-up; ${ }^{* *}$ Re-suppressed at follow-up; ^ Came late $>7$ days for the last drug-refill visit

Table 2: Patterns of reverse transcriptase drug resistance mutations

different clinical guidelines. Here, the frequency of DRM upon virologic failure using a threshold of $>400$ copies $/ \mathrm{mL}$ was similar to thresholds of $\mathrm{VL}>1,000$ or $>5,000$ copies/mL $[14,28,33,42]$ or immunologic failure criteria $(8,15)$. We compared the patterns of DRM found here to 418 published sequences from adult patients failing first-line regimens in subtype C studies, mostly from Southern Africa and India $(8,15,19$, $28,35,37,42)$, accessed, in July 2010 at the Stanford HIV Sequence Database (13). Patterns of DRM, including the overall frequency of any DRM (82\% in the current study vs. $83 \%$ among published sequences), $\geq 1$ NRTI resistance mutations ( $11 \%$ vs $9 \%$ ), the prevalence of $\mathrm{K} 103 \mathrm{~N}$ ( $55 \%$ vs $42 \%$ ), M184V/I ( $66 \%$ vs. $74 \%$ ), and K65R (3\% vs. $6 \%$ ) were not significantly different. However, the data presented here compared to the published sequences demonstrated significant differences in the frequency of $\geq 1$ NNRTI mutation ( $58 \%$ vs $40 \%, p=0.03$ ) and a lower rate of TAM $(21 \%$ vs $37 \%, \mathrm{p}=0.05)$. These modest differences may be ascribed to differences in clinical management strategies, specific drug combinations or duration of virologic failure. Among eight patients with TAMs, the most common pathway (seen in $6 / 8$ patients) was TAM-2 related (D67N, K70R, K219Q/R/E); and the rest (2/8) were mixed with the TAM-1 pathway (M41L, L210W, T215Y) extending similar prior Southern African observations [28,31] .

Although K103N and other NNRTI resistance mutations confer high level NNRTI resistance [12,13], six patients in our study who harbored such mutations, re-suppressed VL after 4 to 10 months with no change in their first-line regimen. Three of these patients had high level resistance to two drug classes, with the addition of the M184V mutation, conferring resistance to lamivudine. These findings extend observations by Hoffmann and colleagues who reported 11 males with either NNRTI and NRTI mutations, who re-suppressed with continued first-line regimens, raising questions about potential cautious re-use or continuation of those medications in certain circumstances [14] . It is plausible that improved adherence was a factor in this observed resuppression, though this could not be confirmed in this patient population. The observation that successful re-suppression was strongly associated with recent failure and a low number of DRM seems logical, but should be confirmed prospectively in larger studies.

Thirteen patients found to be viremic both at enrollment and at 
Citation: El-Khatib Z, DeLong AK, Katzenstein D, Ekstrom AM, Ledwaba J, et al. (2011) Drug Resistance Patterns and Virus Re-Suppression among HIV-1 Subtype C Infected Patients Receiving Non-Nucleoside Reverse Transcriptase Inhibitors in South Africa. J AIDS Clinic Res 2:117. doi:10.4172/2155-6113.1000117

Page 6 of 11

\begin{tabular}{|c|c|c|c|c|}
\hline \multirow[b]{2}{*}{ Risk factor } & \multicolumn{2}{|c|}{$\begin{array}{c}\text { Number of DRM } \\
\text { at study enrollment } \\
(n=43)\end{array}$} & \multicolumn{2}{|c|}{$\begin{array}{l}\begin{array}{l}\text { Persistent virologic failure }(n=13) \text { vs. re-suppression }(n=12) \text { at } \\
\text { follow-up }\end{array}\end{array}$} \\
\hline & $\begin{array}{l}\text { Odds ratio } \\
(95 \% \mathrm{Cl})^{\mathrm{a}}\end{array}$ & p-value & Effect (difference in median, $95 \% \mathrm{Cl} 95 \% \mathrm{Cl}$ ) & $\mathrm{p}$-value \\
\hline Female vs. Male & $5.6(1.3,24.5)$ & 0.02 & $1.1(0.1,10.5)$ & 1.00 \\
\hline Age (years) & $1.0(0.9,1.0)$ & 0.17 & $1.4(-6.0,9.0)$ & 0.62 \\
\hline Current TB therapy $(\text { yes } / \mathrm{no})^{\mathrm{C}}$ & - & - & $0.9(0,78.4)$ & 1.00 \\
\hline Tested HIV positive after 2003 (yes/no) & $0.4(0.1,1.8)$ & 0.23 & $0.7(0.1,5.5)$ & 1.00 \\
\hline Number of months on ART & $1.0(0.9,1.0)$ & 0.16 & $2.1(-1.4,6.4)$ & 0.17 \\
\hline ARV exposure prior to ART initiation ${ }^{d}$ & & - & & 0.78 \\
\hline none & & & reference & \\
\hline pMTCT & -- & --- & $0.3(0,4.3)$ & 0.59 \\
\hline Other ARV & --- & --- & $0.8(0,70.5)$ & 1.00 \\
\hline $\begin{array}{l}\text { Exposure to sdNVP or other ART vs. no } \\
\text { exposure }^{d}\end{array}$ & $3.8(1.1,14.2)$ & 0.03 & $0.42(0,3.8)$ & 0.64 \\
\hline CD4 at study enrollment per 100 cells $/ \mathrm{mm}^{3}$ & $1.7(1.1,2.7)$ & 0.02 & $-0.7(-1.9,1.0)$ & 0.41 \\
\hline CD4 pre-ART initiation per 100 cells $/ \mathrm{mm}^{3}$ & $0.8(0.3,2.1)$ & 0.64 & $0(-0.6,0.5)$ & 0.98 \\
\hline $\log _{10}(V L)$ at study enrollment ${ }^{e}$ & $0.5(0.2,1.0)$ & 0.04 & $0.4(-0.2,1.6)$ & 0.27 \\
\hline $\log _{10}(\mathrm{VL})$ pre-ART initiation ${ }^{\mathrm{e}}$ & $1.3(0.5,3.4)$ & 0.60 & $-0.4(-1.0,0.2)$ & 0.22 \\
\hline $\begin{array}{l}\mathrm{VL}>400 \text { copies } / \mathrm{mL} \text { at least once in the two } \\
\text { tests prior to study enrollment (yes } / \mathrm{no})^{\mathrm{e}}\end{array}$ & $8.4(1.9,42.4)$ & $<0.01$ & (2.4, infinity) & $<0.01$ \\
\hline $\begin{array}{l}\text { Late to the drug refill visit at least } 7 \text { days in } \\
\text { previous month (yes } / \text { no) }\end{array}$ & $0.1(0,0.5)$ & 0.01 & $0.9(0.1,8.6)$ & 1.00 \\
\hline $\begin{array}{l}\text { History of treatment interruption in the last } 6 \\
\text { months (yes/no) }\end{array}$ & --- & --- & (0.2, infinity) & 0.48 \\
\hline Taking d4T vs. AZT & $0.6(0.2,1.9)$ & 0.36 & $0.3(0,2.8)$ & 0.38 \\
\hline Taking EFV vs. NVP & --- & --- & $1.1(0.1,17.8)$ & 1.00 \\
\hline$\geq 1$ DRM (yes/no) & & & $5.1(0.3,313.7)$ & 0.27 \\
\hline Number of NRTI DRM mutations & & & $3.7(1.1,12.2)$ & 0.05 \\
\hline Number of NNRTI DRM mutations & & & $2.9(0.9,9.0)$ & 0.08 \\
\hline Total number of DRM mutations & & & $2.4(1.1,5.0)$ & 0.04 \\
\hline
\end{tabular}

NRTI, nucleoside reverse transcriptase inhibitor; NNRTI, non-NRTI; VL, virus load; TB, tuberculosis; DRM, drug resistance mutations; d4T, stavudine; AZT, zidovudine; EFV, efavirenz; NVP, nevirapine; ARV, antiretroviral drugs

aFor each 1-unit difference in the covariate (e.g. going from no to yes) the odds ratio expresses (for each possible value of the \# DRM (0-7)) the ratio of the odds of having at least that number of DRM versus fewer than that number of DRM.

${ }^{b}$ Effects presented as the odds ratio for categorical risk factors and the difference in medians for continuous risk factors (difference in median, $95 \% \mathrm{Cl}$ ).

'The ordinal logistic regression model could not be fitted because there was too little variability in the risk factor or the assumption of proportional odds ratios was not met (i.e. only 4 were on TB therapy, 4 had taken other ARV prior to starting ART, 3 had treatment interruption, and the assumption was not met for EFV vs. NVP.

¿One female was exposed to both of sdNVP and other ARV, pre-ART initiation, and she had VL $<400$ copies/mL at study enrollment

eVL done using Versant HIV-1 RNA 3.0 (Siemens Deerfield, IL, USA) bDNA technology

Table 3: Risk factors associated with the number of DRM at study enrollment and persistent virologic failure versus re-suppression at follow-up

follow-up, had a longer duration of virologic failure on treatment and a higher number of DRM. These findings substantiate the observation that resistance evolves as a function of continued, albeit suboptimal, drug pressure due to reduced adherence, treatment interruptions or both. Despite the relatively short time between sequences - 8 months, and a median of 3 DRM per patient, mutations accumulated with a rise in high level predicted 2-class resistance. This is consistent with observations in HIV-1 subtype B [5,18]. Some of the accumulated DRM were associated with etravirine resistance, conferring intermediate resistance to this drug after first-line regimen failure, suggesting the need for further studies of the use of this NNRTI in subsequent regimens [23].

Among women, we found a borderline association between exposure to sdNVP and virologic failure, albeit years afterwards and with a period of suppression, consistent with the results of a recent study from the Western Cape, South Africa [6] and another report of a significant association between detection of minority NNRTI mutations and treatment failure, even after 18 months had elapsed since sdNVP [4] . These findings are not entirely in line with other reports 
Citation: El-Khatib Z, DeLong AK, Katzenstein D, Ekstrom AM, Ledwaba J, et al. (2011) Drug Resistance Patterns and Virus Re-Suppression among HIV-1 Subtype C Infected Patients Receiving Non-Nucleoside Reverse Transcriptase Inhibitors in South Africa. J AIDS Clinic Res 2:117. doi:10.4172/2155-6113.1000117

\begin{tabular}{|c|c|c|c|c|c|c|c|c|c|c|c|}
\hline \multirow[b]{3}{*}{$\begin{array}{l}\text { Patien } \\
\mathrm{t}\end{array}$} & \multicolumn{5}{|c|}{ Study enrollment } & \multicolumn{6}{|c|}{ Follow-up } \\
\hline & \multirow[b]{2}{*}{$\begin{array}{l}\text { VL } \\
\text { (ART } \\
\text { used)* }\end{array}$} & \multirow[b]{2}{*}{$\begin{array}{l}\text { NNRTI } \\
\text { mutations }\end{array}$} & \multirow[b]{2}{*}{$\begin{array}{l}\text { NRTI } \\
\text { mutatio } \\
\text { ns }\end{array}$} & \multicolumn{2}{|c|}{$\begin{array}{l}\text { Predicted phenotypic } \\
\text { resistance }\end{array}$} & \multirow[b]{2}{*}{$\begin{array}{l}\text { Months } \\
\text { after } \\
\text { enroll } \\
\text { ment } \\
\end{array}$} & \multirow[b]{2}{*}{$\begin{array}{l}\text { VL } \\
\text { (ART } \\
\text { used)* }\end{array}$} & \multirow[b]{2}{*}{$\begin{array}{l}\text { NNRTI } \\
\text { mutations }\end{array}$} & \multirow[b]{2}{*}{$\begin{array}{l}\text { NRTI } \\
\text { mutations }\end{array}$} & \multicolumn{2}{|c|}{$\begin{array}{l}\text { Predicted phenotypic } \\
\text { resistance }\end{array}$} \\
\hline & & & & NNRTI & NRTI & & & & & NNRTI & NRTI \\
\hline 1 & $\begin{array}{l}3,010 \\
(1 \mathrm{~b})\end{array}$ & $\begin{array}{l}\text { K101E+ } \\
\text { V1081+ } \\
\text { Y181C }\end{array}$ & $\begin{array}{l}\text { D67N+ } \\
\text { K70R+ } \\
\text { M184V } \\
+ \\
\text { K219E }\end{array}$ & $\begin{array}{l}\text { H: NVP } \\
\text { I: EFV; } \\
\text { ETR }\end{array}$ & $\begin{array}{l}\text { H: 3TC } \\
\text { l: AZT } \\
\text { L: d4T; ABC } \\
\text { PL: ddl } \\
\text { S: TDF }\end{array}$ & 7 & $\begin{array}{l}3,680 \\
(1 \mathrm{~b})\end{array}$ & $\begin{array}{l}\text { K101E+ } \\
\text { Y181C }\end{array}$ & $\begin{array}{l}\text { D67N+ } \\
\text { K70R+ } \\
\text { M184V+ } \\
\text { K219E }\end{array}$ & $\begin{array}{c}\text { H: NVP } \\
\text { I: EFV } \\
\text { ETR }\end{array}$ & $\begin{array}{l}\text { H: 3TC } \\
\text { I: AZT } \\
\text { L: d4T; ABC } \\
\text { PL: ddl } \\
\text { S: TDF }\end{array}$ \\
\hline 3 & $\begin{array}{l}4,310 \\
\text { (1a) }\end{array}$ & $\begin{array}{l}\text { K101E+ } \\
\text { V106M+ } \\
\text { G190A }\end{array}$ & $\begin{array}{l}\text { D67N+ } \\
\text { M184V }\end{array}$ & $\begin{array}{l}\text { H: EFV; } \\
\text { NVP } \\
\text { l: ETR }\end{array}$ & $\begin{array}{l}\text { H: 3TC } \\
\text { L: ABC } \\
\text { PL: ddl } \\
\text { S: d4T; AZT } \\
\text { TDF }\end{array}$ & 11 & $\begin{array}{l}3,790 \\
(1 a)\end{array}$ & $\begin{array}{l}\text { K101E+ } \\
\text { V106M }+ \\
\text { G190A }\end{array}$ & $\begin{array}{l}\text { D67N+ } \\
\text { K70R+ } \\
\text { M184V+ } \\
\text { K219Q }\end{array}$ & $\begin{array}{l}\text { H: EFV; NVP } \\
\text { l: ETR }\end{array}$ & $\begin{array}{l}\text { H: 3TC } \\
\text { l: AZT } \\
\text { L: d4T; ABC } \\
\text { PL: ddl } \\
\text { S: TDF }\end{array}$ \\
\hline 5 & $\begin{array}{l}1,850 \\
(1 a)\end{array}$ & $\begin{array}{l}\text { K103N+ } \\
\text { V108I }\end{array}$ & $\begin{array}{l}\text { A62V+ } \\
\text { V75I+ } \\
\text { M184V }\end{array}$ & $\begin{array}{l}\text { H: EFV; } \\
\text { NVP } \\
\text { S: ETR }\end{array}$ & $\begin{array}{l}\text { H: } 3 T C \\
\text { L: ddl; ABC } \\
\text { S: d4T; AZT } \\
\quad \text { TDF }\end{array}$ & 6 & $\begin{array}{l}6,150 \\
\text { (1a) }\end{array}$ & $\begin{array}{l}\text { K103N+ } \\
\text { V108I }\end{array}$ & $\begin{array}{l}\text { A62V+ } \\
\text { V75I+ } \\
\text { M184V }\end{array}$ & $\begin{array}{l}\text { H: EFV; NVP } \\
\text { PL: ETR }\end{array}$ & $\begin{array}{l}\text { H: 3TC } \\
\text { L: ddl; ABC } \\
\text { S: d4T; TDF } \\
\text { AZT }\end{array}$ \\
\hline 6 & $\begin{array}{l}773 \\
\text { (1a) }\end{array}$ & $\begin{array}{l}\text { V106M+ } \\
\text { G190A+ } \\
\text { A98G }\end{array}$ & $\begin{array}{l}\text { M41L } \\
\text { D67N+ }+ \\
\text { K70R+ } \\
\text { M184V }\end{array}$ & $\begin{array}{l}\text { H: EFV; } \\
\text { NVP } \\
\text { L: ETR }\end{array}$ & $\begin{array}{l}\text { H: } 3 T C \\
\text { I: } d 4 T, A B C \\
\text { AZT } \\
\text { L: TDF } \\
\text { ddl }\end{array}$ & 9 & $\begin{array}{l}4,530 \\
(1 a)\end{array}$ & $\begin{array}{l}\text { A98G+ } \\
\text { V106M+ } \\
\text { G190A }\end{array}$ & $\begin{array}{l}\text { D67N+ } \\
\text { K70R+ } \\
\text { M184V+ } \\
\text { K219Q }\end{array}$ & $\begin{array}{l}\text { H: EFV; NVP } \\
\text { L: ETR }\end{array}$ & $\begin{array}{l}\text { H: 3TC } \\
\text { I: AZT } \\
\text { L: d4T } \\
\text { ABC } \\
\text { PL: ddl } \\
\text { S: TDF }\end{array}$ \\
\hline 7 & $\begin{array}{l}2,530 \\
\text { (1a) }\end{array}$ & $\begin{array}{l}\text { V106M+ } \\
\text { Y188L므 }\end{array}$ & $\begin{array}{l}\mathrm{D} 67 \mathrm{~N}+ \\
\mathrm{M} 184 \mathrm{~V} \\
+ \\
\mathrm{K} 219 \mathrm{E} \\
\end{array}$ & $\begin{array}{l}\text { H: EFV; } \\
\text { NVP } \\
\text { L: ETR }\end{array}$ & $\begin{array}{l}\text { H: } 3 T C \\
\text { L: d4T; ABC } \\
\text { AZT } \\
\text { PL: ddl } \\
\text { S: TDF }\end{array}$ & 12 & $\begin{array}{l}25,000 \\
(1 a)\end{array}$ & $\begin{array}{l}\text { V90I+ } \\
\text { V106M+ } \\
\text { Y188L }\end{array}$ & M184V & $\begin{array}{l}\text { H: EFV; NVP } \\
\text { L: ETR }\end{array}$ & $\begin{array}{l}\text { H: } 3 T C \\
\text { PL: ABC } \\
\text { S: d4T; TDF } \\
\text { ddl; AZT }\end{array}$ \\
\hline 12 & $\begin{array}{l}49,900 \\
(1 a)\end{array}$ & $\begin{array}{l}\text { K103N+ } \\
\text { Y181C+ } \\
\text { P225H }\end{array}$ & M184I & $\begin{array}{l}\text { H: EFV; } \\
\text { NVP } \\
\text { l: ETR }\end{array}$ & $\begin{array}{l}\text { H: } 3 T C \\
\text { PL: ABC } \\
\text { S: d4T; TDF } \\
\quad \text { AZT }\end{array}$ & 6 & $\begin{array}{l}8,540 \\
\text { (1a) }\end{array}$ & $\begin{array}{l}\text { V90l+ } \\
\text { K103N+ } \\
\text { Y181C+ } \\
\text { P225H }\end{array}$ & M184V & $\begin{array}{l}\text { H: EFV; NVP } \\
\text { l: ETR }\end{array}$ & $\begin{array}{l}\text { H: 3TC } \\
\text { PL: ABC } \\
\text { S: d4T; TDF } \\
\text { AZT; ddl }\end{array}$ \\
\hline 15 & $\begin{array}{l}18,300 \\
(1 a)\end{array}$ & $\begin{array}{l}\text { K101E+ } \\
\text { Y188L }\end{array}$ & M184I & $\begin{array}{l}\text { H: EFV; } \\
\text { NVP } \\
\text { L: ETR }\end{array}$ & $\begin{array}{l}\text { H: 3TC } \\
\text { PL: ABC } \\
\text { S: d4T; TDF } \\
\text { ddl; AZT }\end{array}$ & 9 & $\begin{array}{l}3,710 \\
(1 a)\end{array}$ & $\begin{array}{l}\text { K101E+ } \\
\text { Y188L }\end{array}$ & M184V & $\begin{array}{l}\text { H: EFV; NVP } \\
\text { L: ETR }\end{array}$ & $\begin{array}{l}\text { H: 3TC } \\
\text { PL: ABC } \\
\text { S: d4T; TDF } \\
\text { ddl; AZT }\end{array}$ \\
\hline 18 & $\begin{array}{l}407 \\
(1 a)\end{array}$ & $\begin{array}{l}\mathrm{K} 103 \mathrm{~N}+ \\
\mathrm{P} 225 \mathrm{H}\end{array}$ & M184V & $\begin{array}{l}\text { H: EFV; } \\
\text { NVP } \\
\text { L: ETR }\end{array}$ & $\begin{array}{l}\mathrm{H}: 3 T C \\
\text { PL: ABC } \\
\text { S: d4T; TDF } \\
\text { DDI; AZT }\end{array}$ & 10 & $\begin{array}{l}937 \\
(1 \mathrm{~b})\end{array}$ & K103N & M184V & $\begin{array}{l}\text { H: EFV, NVP } \\
\text { S: ETR }\end{array}$ & $\begin{array}{l}\text { H: 3TC } \\
\text { PL: ABC } \\
\text { S: d4T; TDF } \\
\text { ddl; AZT }\end{array}$ \\
\hline 20 & $\begin{array}{l}1,330 \\
(1 a)\end{array}$ & $\begin{array}{l}\text { K103N+ } \\
\text { V108I }\end{array}$ & M184V & $\begin{array}{l}\text { H: EFV; } \\
\text { NVP } \\
\text { S: ETR }\end{array}$ & $\begin{array}{l}\text { H: 3TC } \\
\text { PL: ABC } \\
\text { S: d4T; DDI } \\
\text { TDF; AZT }\end{array}$ & 10 & $\begin{array}{l}3,010 \\
(1 a)\end{array}$ & $\begin{array}{l}\text { K103N+ } \\
\text { V108I }\end{array}$ & M184V & $\begin{array}{l}\text { H: EFV; NVP } \\
\text { S: ETR }\end{array}$ & $\begin{array}{l}\text { H: 3TC } \\
\text { PL: ABC } \\
\text { S: d4T; TDF } \\
\text { ddl; AZT }\end{array}$ \\
\hline 23 & $\begin{array}{l}955 \\
(1 \mathrm{~b})\end{array}$ & V106M & $\begin{array}{l}\mathrm{A} 62 \mathrm{~V}+ \\
\mathrm{M} 184 \mathrm{~V}\end{array}$ & $\begin{array}{l}\text { H: EFV; } \\
\text { NVP } \\
\text { PL: } \\
\text { ETR }\end{array}$ & $\begin{array}{l}\text { H: 3TC } \\
\text { PL: ABC } \\
\text { S: d4T; TDF } \\
\text { DDI; AZT }\end{array}$ & 11 & $\begin{array}{l}2,540 \\
(1 \mathrm{~b})\end{array}$ & $\begin{array}{l}\text { V106M+ } \\
\text { E138A }\end{array}$ & $\begin{array}{l}\mathrm{A} 62 \mathrm{~V}+ \\
\mathrm{M} 184 \mathrm{~V}\end{array}$ & $\begin{array}{l}\text { H: EFV; NVP } \\
\text { L: ETR }\end{array}$ & $\begin{array}{l}\text { H: 3TC } \\
\text { PL: ABC } \\
\text { S: d4T; TDF } \\
\text { ddl; AZT }\end{array}$ \\
\hline 30 & $\begin{array}{l}1,130 \\
(1 a)\end{array}$ & $\mathrm{K} 103 \mathrm{~N}$ & --- & $\begin{array}{l}\text { H: EFV; } \\
\text { NVP } \\
\text { S: ETR }\end{array}$ & S: all drugs & 9 & $\begin{array}{l}15,700 \\
(1 a)\end{array}$ & K103N & V75I & $\begin{array}{l}\text { H: EFV; NVP } \\
\text { S: ETR }\end{array}$ & $\begin{array}{l}\text { PL: d4T; ddl } \\
\text { S: 3TC; TDF } \\
\text { ABC; } \\
\text { AZT }\end{array}$ \\
\hline 35 & $\begin{array}{l}83,000 \\
(1 a)\end{array}$ & Wild type & $\begin{array}{l}\text { Wild } \\
\text { type }\end{array}$ & $\begin{array}{l}\text { S: all } \\
\text { drugs }\end{array}$ & S: all drugs & 9 & $\begin{array}{l}2,670 \\
(1 a)\end{array}$ & V106M & $\begin{array}{l}\text { K70R+ } \\
\text { M184V }\end{array}$ & $\begin{array}{l}\text { H: EFV; NVP } \\
\text { PL: ETR }\end{array}$ & $\begin{array}{l}\text { H: 3TC } \\
\text { PL: ABC; } \\
\text { AZT } \\
\text { S: d4T; } \\
\text { TDF; } \\
\quad \text { ddl }\end{array}$ \\
\hline 40 & 4660 & $\begin{array}{l}\text { Not } \\
\text { amplifiable }\end{array}$ & $\begin{array}{l}\text { Not } \\
\text { amplifia } \\
\text { ble }\end{array}$ & --- & --- & 9 & 31,300 & $\begin{array}{l}\text { K103N+ } \\
\text { P225H }\end{array}$ & $\begin{array}{l}\text { V75I+ } \\
\text { M184V }\end{array}$ & $\begin{array}{l}\text { H: EFV; NVP } \\
\text { L: ETR }\end{array}$ & $\begin{array}{l}\text { H: 3TC } \\
\text { L: ABC; DDI } \\
\text { S: d4T; } \\
\text { AZT; } \\
\quad \text { TDF }\end{array}$ \\
\hline
\end{tabular}

NRTI, nucleoside reverse transcriptase inhibitor: NNRTI, non-NRTI: VL, virus load (copies/mL); ART, antiretroviral therapy; EFV, Efavirenz; NVP, Nevirapine; ETR, Etravirine; 3TC, Lamivudine; d4T, Stavudine; TDF, Tenofovir; ABC, Abacavir; AZT, Zidovudine; DDI, Didanosine; S, Susceptible; PL, potentially low-level of resistance; L, low-level resistance; I, intermediate level of resistance; H, High level of resistance; Line 1a, EFV + 3TC + d4T; Line 1b, NVP + 3TC + d4T

Underlined mutations = lost mutations; Bolded mutations=gained mutations and Italic mutations=Minor mutations, for Etravirine, according to the IAS USA-2009 list. Background colors for predicted phenotypic resistance were: White if susceptible or potentially-low; Light gray if low or intermediate and dark gray if highly resistant.

Table 4a: Genotypic and predicted phenotypic drug resistance among patients with persistent virologic failure at follow-up 
Citation: El-Khatib Z, DeLong AK, Katzenstein D, Ekstrom AM, Ledwaba J, et al. (2011) Drug Resistance Patterns and Virus Re-Suppression among HIV-1 Subtype C Infected Patients Receiving Non-Nucleoside Reverse Transcriptase Inhibitors in South Africa. J AIDS Clinic Res 2:117. doi:10.4172/2155-6113.1000117

Page 8 of 11

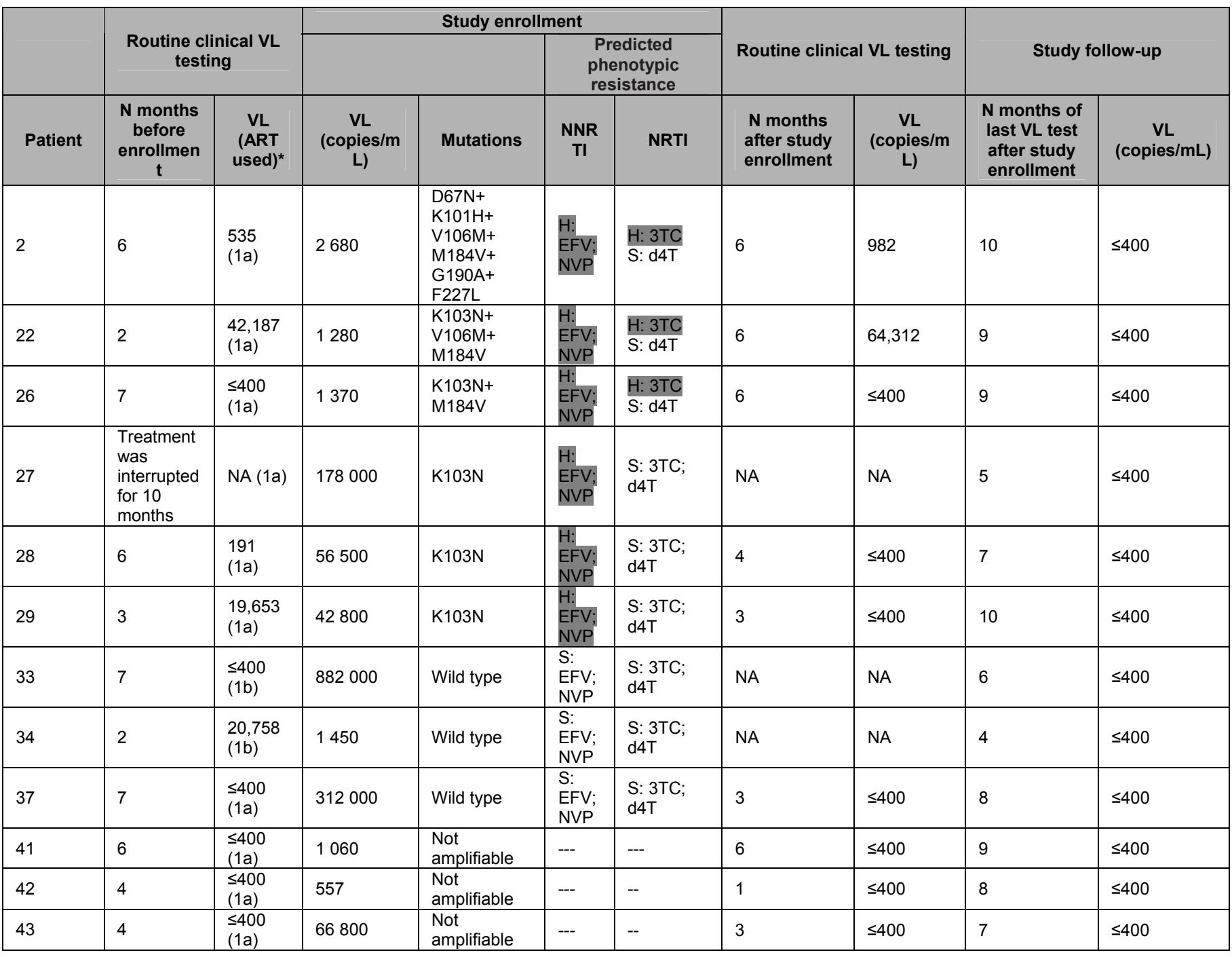

NRTI, nucleoside reverse transcriptase inhibitor; NNRTI, non-NRTI; VL, virus load (copies/mL); TB, tuberculosis; DRM, drug resistance mutations; Line 1a, Efavirenz (EFV) + Lamivudine (3TC) + Stavudine (d4T); Line 1b, Nevirapine (NVP) + 3TC + d4T; Colors for predicted phenotypic resistance were: White if susceptible (S) or potentially-low $(\mathrm{PL})$; Light gray if low $(\mathrm{L})$ or intermediate $(\mathrm{I})$ and dark gray if highly $(\mathrm{H})$ resistant.

Table 4b: Characteristics of patients with virologic failure at study enrollment and re-suppression at follow-up

from sub-Saharan Africa and Asia that suggest that in the short-term, administration of ART $>12$ months after sdNVP may not jeopardize the efficacy of NNRTI based ART [17,26].

Where genotyping is routinely available, the interpretation of DRM following the failure of first-line regimens drives decisions about clinical management, adherence counseling, switching to second-line regimens and the need for new ART combinations. Among these HIV-1 subtype C infected patients, a proportion of those failing firstline regimens responded to continuing their current regimen despite predicted high-level resistance. In resource-limited settings, where genotyping is not available, the practice of reinforcing adherence, continuing a first-line regimen and repeating a VL test after 6 months may be justified. However, it will be important to monitor such patients closely to determine if they ultimately fail due to the presence of archived resistance mutations. In such cases, switching to a second-line regimen, as mandated by treatment national guidelines, should not be deferred.

There are several limitations to this study. First, the number of patients with drug resistance, longitudinal sequences and re-suppression was relatively small and longer term CD4 cell counts and VLs from 25 failing patients were not available. Second, a small proportion of patients' samples, particularly among those who resuppressed, could not be successfully amplified and sequenced. Third, genotyping at onset of ART was not available. However, the prevalence of transmitted ART resistance, in South Africa, is still low $(<5 \%)[24,34]$. Finally, confirmation of VL was not immediately available, and clinical practice included continued first line-treatment for 4-6 months between detection of virologic failure, reinforcing adherence and re-testing VL. The fact that patients were not switched to second-line regimens, despite repeated virologic failure, reflects the current limited treatment options in this setting.

In summary, this study extends observations of the range of DRM patterns among HIV-1 subtype C patients in South Africa receiving long-term first-line regimens. Surprisingly, a number of patients with DRM that predict high-level resistance, K103N with or without M184V, were successfully re-suppressed on the same first-line regimen. Reinforcing adherence without changing treatment among 
Citation: El-Khatib Z, DeLong AK, Katzenstein D, Ekstrom AM, Ledwaba J, et al. (2011) Drug Resistance Patterns and Virus Re-Suppression among HIV-1 Subtype C Infected Patients Receiving Non-Nucleoside Reverse Transcriptase Inhibitors in South Africa. J AIDS Clinic Res 2:117. doi:10.4172/2155-6113.1000117

Page 9 of 11

patients with first-line virologic failure could spare the expense and toxicity of second-line regimens in resource-limited settings with high HIV burdens. While additional studies are needed to confirm these observations and examine their longevity, detection of viremia on treatment should prompt repeat testing, adherence counseling and, if viremia is persistent, provision of second-line therapy.

\section{Acknowledgments}

We would like to thank patients for agreeing to participate, Ms. Albertina Dambuza and Belinda Dambuza for assisting in reviewing medical records; Sisters Noreen Boikanyo, Rebecca Phofa, Catherine Lephoto, Agnes Ramashiga and Fikile Mbatha for study logistics; Ms Sarah Cohen, Mary Phoswa and Maphut Madiga for processing blood specimens; Ms Ewalde Cutler and Professor Adrian Puren for the VL and CD4 cell counts; Drs. Penny Moore for technical assistance and Robert Shafer for constructive critiques.

This study was funded by: the Swedish International Development Cooperation Agency to Z.EK and NICD; Sven Gard's Fund for Virology Research, The Roya Swedish Academy of Sciences and a Karolinska Institutet Faculty Funds (KID) to Z.EK; African Programme for Training in HIV/TB Research Fogarty International Center/NIH/2U2RTW006878 and from the Freeman-Spogli Institute at Stanford University; D.K, A.DL and R.K are funded by an NIH/RO1 grant/AI66922. A.DL is also funded by the National Institute Of Allergy And Infectious Diseases/ P30AI042853.

\section{Gen Bank accession number: HQ12279-12308;HQ12354-12365.}

Competing Interests: The authors have declared that no competing interests exist

\section{References}

1. Bangsberg DR, Kroetz DL, Deeks SG (2007) Adherence-resistance relationships to combination HIV antiretroviral therapy. Curr HIVIAIDS Rep 4 : $65-72$

2. Barth RE, van der Loeff MF, Schuurman R, Hoepelman Al, Wensing AM (2010) Virological follow-up of adult patients in antiretroviral treatment programmes in sub-Saharan Africa: a systematic review. Lancet Infect Dis 10:155-166.

3. Charalambous SA, Grant JD, Pemba L, Chaisson R, Kruger P, et al. (2007) Establishing a workplace antiretroviral therapy programme in South Africa. AIDS Care 19: 34-41.

4. Coovadia A, Hunt G, Abrams EJ, Sherman G, Meyers T et al. (2009) Persistent minority $\mathrm{K} 103 \mathrm{~N}$ mutations among women exposed to single-dose nevirapine and virologic response to Nonnucleoside Reverse-Transcriptase Inhibitorbased therapy. Clin Infect Dis 48: 462-472.

5. Cozzi-Lepri A, Phillips AN, Ruiz L, Clote B , Loveday C, et al. (2007) Evolution of drug resistance in HIV-infected patients remaining on a virologically failing combination antiretroviral therapy regimen. AIDS 21:721-732.

6. Datay MI, Boulle A, Mant D,Yudkin P (2010) Associations with virologic treatment failure in adults on antiretroviral therapy in South Africa. J Acquir Immune Defic Syndr 54: 489-495.

7. de Oliveira T, Deforche K, Cassol S, Salminen M, Paraskevis D, et al. (2005) An automated genotyping system for analysis of HIV-1 and other microbial sequences. Bioinformatics 21:3797-800.

8. Deshpande A, Jeannot AC, Schrive MH, Wittkop L, Pinson P, et al. (2010) Analysis of RT sequences of subtype C HIV-type 1 isolates from Indian patients at failure of a first-line treatment according to clinical and/or immunological WHO guidelines. AIDS Res Hum Retrovirus 26: 343-350.

9. El-Khatib Z (2004) Questionnaire design and data entry in EpiData Guideline. Division of Clinical Cancer Epidemiology - Karolinska Institutet.

10. El-Khatib ZA, Ekstrom M, Laher F, Karstaedt AS, Petzold M, et al. (2009) Medical review-South African adherence and virologic evaluation (MR. SAVE) tool, 1.0 ed.

11. El-Khatib Z, Ekstrom AM, Ledwaba J, Mohapi L, Laher F, et al. (2010) Viremia and drug resistance among HIV-1 patients on antiretroviral treatment: a crosssectional study in Soweto, South Africa. AIDS 24: 1679-1687.

12. Hammer SM, Eron JJ, Reiss P, Schooley RT, Thompson MA, et al. (2008) Antiretroviral treatment of adult HIV infection: 2008 recommendations of the
International AIDS Society-USA panel. JAMA 300: 555-570.

13. HIV Stanford Database (2010) HIV Stanford Database , vol. 2010, Palo Alto California, USA.

14. Hoffmann CJ, Charalambous S, Sim J, Ledwaba J, Schwikkard G,et al. (2009) Viremia, resuppression, and time to resistance in Human Immunodeficiency Virus (HIV) Subtype C during first-line antiretroviral therapy in South Africa. Clin Infect Dis 49:1928-1935.

15. Hosseinipour MC, van Oosterhout JJ, Weigel R, Phiri S, Kamwendo D, et al (2009) The public health approach to identify antiretroviral therapy failure: highlevel nucleoside reverse transcriptase inhibitor resistance among Malawians failing first-line antiretroviral therapy. AIDS 23: 1127-1134.

16. Johnson, VA, Brun-Vezinet F, Clotet B, Gunthard HF, Kuritzkes DR, et al. (2009) Update of the drug resistance mutations in HIV-1. Top HIV Med 17:138-145.

17. Jourdain G, Ngo-Giang-Huong N, Le Coeur S, Bowonwatanuwong C Kantipong $P$, et al. (2007) Intrapartum exposure to nevirapine and subsequent maternal responses to nevirapine-based antiretroviral therapy. N Engl J Med 356: 135-147.

18. Kantor R., Shafer RW, Follansbee S, Taylor J, Shilane D,et al. (2004) Evolution of resistance to drugs in HIV-1-infected patients failing antiretroviral therapy. AIDS 18: 1503-1511.

19. Kassaye S, Johnston E, McColgan B, Kantor R, Zijenah L, et al. (2009) Envelope coreceptor tropism, drug resistance, and viral evolution among subtype C HIV-1-infected individuals receiving nonsuppressive antiretroviral therapy. J Acquir Immune Defic Syndr 50: 9-18.

20. Keiser O, Orrell C, Egger M, Wood R, Brinkhof MW, et al. (2008) Public-health and individual approaches to antiretroviral therapy: Township South Africa and Switzerland compared. PLoS Med 5:e148.

21. Kumarasamy NV, Madhavan K Venkatesh S, Saravanan R, Kantor $P$, et al (2009) High frequency of clinically significant mutations after first-line generic highly active antiretroviral therapy failure: implications for second-line options in resource-limited settings. Clin Infect Dis 49: 306-309.

22. Lallemant M, Jourdain G, Le Coeur S, Mary JY, Ngo-Giang-Huong N, et al. (2004) Single-dose perinatal nevirapine plus standard zidovudine to prevent mother-to-child transmission of HIV-1 in Thailand. N Engl J Med 351: 217-228.

23. Lazzarin A, Campbell T, Clotet B, Johnson M, Katlama C, et al. (2007) Efficacy and safety of TMC125 (etravirine) in treatment-experienced HIV-1-infected patients in DUET-2: 24-week results from a randomised, double-blind, placebocontrolled trial. Lancet 370: 39-48.

24. Ledwaba J, Hunt G, Rakgotho M, El-Khatib Z, Singh S, et al. (2009) HIV-1 transmitted drug resistance surveillance in three provinces in South Africa during 2002-2007. Antivir Ther 14: A182.

25. Lockman S (2008) Prevention of mother-to-child transmission, drug resistance, and implications for response to therapy. Curr Opin HIV AIDS 3:166-72.

26. Lockman S, Shapiro RL, Smeaton LM, Wester C, Thior I, et al. (2007) Response to antiretroviral therapy after a single, peripartum dose of nevirapine. $\mathrm{N}$ Engl J Med 356:135-147

27. Maartens G, Venter F, Meintjes G, Cohen K (2008) Southern African HIV clinicians society - Guidelines for antiretroviral therapy in adults. 03-Oct.-2009.

28. Marconi VC, Sunpath H, Lu Z, Gordon M, Koranteng-Apeagyei K, et al (2008) Prevalence of HIV-1 drug resistance after failure of a first highly active antiretroviral therapy regimen in KwaZulu Natal, South Africa. Clin Infect Dis 46:1589-1597.

29. Martinson N, Mohapi L, Bakos D, Gray GE, McIntyre JA, et al. (2009) Costs of providing care for HIV-infected adults in an urban HIV clinic in Soweto, South Africa. J Acquir Immune Defic Syndr 50: 327-330.

30. Nachega JB, Hislop M, Dowdy DW, Chaisson RE, Regensberg L, et al. (2007) Adherence to nonnucleoside reverse transcriptase inhibitor-based HIV therapy and virologic outcomes. Ann Intern Med 146: 564-574.

31. Novitsky V, Wester CW, DeGruttola V, Bussmann H, Gaseitsiwe S, et al. (2007) The reverse transcriptase $67 \mathrm{~N} 70 \mathrm{R} 215 \mathrm{Y}$ genotype is the predominant TAM pathway associated with virologic failure among HIV type 1C-infected adults treated with ZDV/ddl-containing HAART in southern Africa. AIDS Res Hum 
Citation: El-Khatib Z, DeLong AK, Katzenstein D, Ekstrom AM, Ledwaba J, et al. (2011) Drug Resistance Patterns and Virus Re-Suppression among HIV-1 Subtype C Infected Patients Receiving Non-Nucleoside Reverse Transcriptase Inhibitors in South Africa. J AIDS Clinic Res 2:117. doi:10.4172/2155-6113.1000117

Page 10 of 11

Retrovirus 23:868-878.

32. Orrell C, Harling G, Lawn SD, Kaplan R, McNally M, et al. (2007) Conservation of first-line antiretroviral treatment regimen where therapeutic options are limited. Antivir Ther 12: 83-88

33. Orrell C, Walensky RP, Losina E, Pitt J, Freedberg JA, et al. (2009) HIV type1 clade $\mathrm{C}$ resistance genotypes in treatment-naive patients and after first virological failure in a large community antiretroviral therapy programme. Antivir Ther 14: 523-531.

34. Pillay V, Ledwaba J, Hunt G, Rakgotho M, Singh B, et al. (2007) Antiretroviral drug resistance surveillance among drug-naive HIV-1-infected individuals in Gauteng Province, South Africa in 2002 and 2004. Antivir Ther 13: 101-107.

35. Pillay V, Pillay C, Kantor R, Venter F, Levin L, et al. (2008) HIV type 1 subtype $C$ drug resistance among pediatric and adult South African patients failing antiretroviral therapy. AIDS Res Hum Retrovirus 24:1449-1454.

36. Ramadhani HO, Thielman NM, Landman KZ, Ndosi EM, Gao F, et al. (2007) Predictors of incomplete adherence, virologic failure, and antiviral drug resistance among HIV-infected adults receiving antiretroviral therapy in Tanzania. Clin Infect Dis 45: 1492-1498.

37. Sen S, Tripathy SP, Patil AA, Chimanpure VM, R. Paranjape RS (2007) High prevalence of human immunodeficiency virus type 1 drug resistance mutations in antiretroviral treatment-experienced patients from Pune, India. AIDS Res Hum Retrovirus 23:1303-1308.

38. StataCorp LP (2008) Stata/SE 10.1 College Station, TX 77845 USA.

39. Steingrover R, Pogany K, Fernandez Garcia E, Jurriaans S, Brinkman K, et al. (2008) HIV-1 viral rebound dynamics after a single treatment interruption depends on time of initiation of highly active antiretroviral therapy. AIDS 22:1583-1588

40. The R project for statistical computing (2010) The R project for statistical computing

41. Uy J, Armon C, Buchacz K, Wood K, Brooks JT (2009) Initiation of HAART at higher CD4 cell counts is associated with a lower frequency of antiretroviral drug resistance mutations at virologic failure. J Acquir Immune Defic Syndr 51: 450-453.

42. Wallis CL, Mellors JW, Venter WD, Sanne I, Stevens W (2009) Varied patterns of HIV-1 drug resistance on failing first-line antiretroviral therapy in South Africa. J Acquir Immune Defic Syndr 53: 480-484.

43. World Health Organization (WHO) UNAIDS, and UNICEF (2009) Towards universal access - Scaling up priority HIVIAIDS interventions in the health sector. 